\title{
Comparison of Unblocked, Directionally Blocked and Full Blocked Plans in Helical Tomotherapy for Breast Cancer Radiotherapy
}

Eda Kucuktulu1 $^{*}$, Ahmet Fatih Yurekli ${ }^{2}$, Eray Bilcan ${ }^{3}$, Mahmut Serdar Sisecioglu $^{4}$, Murat Topbas ${ }^{5}$ and Uzer Kucuktulu ${ }^{6}$

${ }^{1}$ Consultant, Kanuni Research and Training Hospital, Radiation Oncology, Trabzon, Turkey

${ }^{2}$ TC Ministry of Health Ankara Ataturk Training and Research Hospital, Ankara, Turkey

${ }^{3}$ Adana City Hospital, Adana, Turkey

${ }^{4}$ Inonu University Turgut Ozal Medical Center, Malatya, Turkey

${ }^{5}$ Karadeniz Technical University, Trabzon, Turkey

${ }^{6}$ Trabzon Law Education and Research Hospital, Trabzon, Turkey

\begin{abstract}
Introduction: The doses received by the contralateral breast, lung and heart either directionally or full blocked techniques in Hi-Art Tomotherapy systems in partial or whole breast radiotherapy has been studied.

Method: In 20 breast cancer patients 3 plans with non-blocked, directionally blocked and full blocked methods were designed in Hi-Art planning system. In all planning method the dose received by the contralateral breast, the heart volume percentages V5 and V30 and the lung volume percentages V5 and V20 were compared.

Results: The mean doses received by contralateral breast were $6.24 \mathrm{~Gy}(4.50-8.74 \mathrm{~Gy})$ in unblocked group $3.85 \mathrm{~Gy}(2.08-5.81 \mathrm{~Gy})$ in directionally blocked group and $0.99 \mathrm{~Gy}(0.69-1.39 \mathrm{~Gy})$ in full blocked group. The mean Lung V5 values were $98 \%(79-100 \%)$ in unblocked group and it was $98 \%(91-100 \%)$ in directionally blocked group while it was 93\% (89-97\%) in full blocked group. The mean V20 values were $16 \%(7-23 \%), 20 \%(17-24 \%)$ and $22 \%$ $(18-28 \%)$ in unblocked, directionally blocked and full blocked groups respectively. The mean Heart V5 value $100 \%$ $(99-100 \%)$ in unblocked group, and it was $99 \%(85-100 \%)$ in directionally blocked group while it was $92 \%$ (73$100 \%)$ in full blocked group. The mean V30 values were $11 \%(2-25 \%), 6 \%(0-12 \%)$ and $9 \%(1-22 \%)$ in unblocked, directionally blocked and full blocked groups respectively. The mean beam-on time was $261.6 \mathrm{~s}(237.6-318.2)$ for unblocked, $277.9 \mathrm{~s}$ (237.6-338.5) for directionally blocked and $314.2 \mathrm{~s}(272.6-429.1)$ full blocked group. The mean numbers of MUs were 3712 (3353-4536) for unblocked, 3951 (3357-4834) for directionally blocked and 4506 (38666168) for full blocked group. In full blocked group both beam on time and MU values were higher.
\end{abstract}

Conclusion: We propose that further efforts needed to reduce low dose volumes to normal tissues, meanwhile when using directionally and full blocked plans all parameters should be considered together.

Keywords: Breast carcinoma; Tomotherapy; Intensity modulated radiotherapy

\section{Introduction}

In daily practice, the use of Intensity Modulated Radiotherapy (IMRT) is in an upward tendency following technological developments in the field. When breast or chest wall, additionally supraclavicular field (SCF) and axillary nodes treatment is attempted, a complicated target volume develops in close vicinity with lung, heart and contralateral breast [1]. In such cases, in various IMRT techniques when required dose homogeneity is provided there are variations in doses that normal tissues received. [2] Locoregional breast IMRT has been compared with CT based three dimensional (3D) planning [3-5]. In these studies, IMRT has provided similar target volume coverage and has yielded improvements in dose homogeneity while sparing the lungs and heart from high doses of radiation but again showed an associated increase in integral dose [6].

Helical Tomotherapy (TOMO) which is an IMRT technique, the radiation delivery is provided by a rotating gantry around the patient. In various studies concerning locoregional therapies for breast cancer carried out up to date, increase in volumes receiving from TOMO plans was demonstrated The higher contralateral breast doses were also reported $[7,8]$. In Caudell et al. and Caudrelier et al. studies, despite the use of directional block for lung, heart and contralateral breast, the doses for lung and contralateral breast for TOMO plans were much higher $[7,8]$.

Since the life expectancy of breast cancer patients has increased after modern therapy modalities, the radiation doses received by lung, heart and contralateral breast tissue became more important. The concerns have risen concerning the risk of second cancer in tissues after low dose irradiation. [9]. It is very important to investigate the volumes of normal tissues receiving low dose irradiation. We studied the doses received by the contralateral breast; lung and heart either directionally or full blocked techniques in Hi-Art tomotherapy system which is recently popularized in partial or whole breast radiotherapy.

\section{Materials and Methods}

\section{Patient's selection, CT simulation and target delineation}

The mean age of our 20 patients was 51 years (38-80), nine of which had tumors of the right breast while 11 patients had left breast tumors. All patients were at Stage II/III. Four patients had breast conserving

*Corresponding author: Eda Kucuktulu, Consultant, Kanuni Research and Training Hospital, Radiation Oncology, Trabzon, Turkey, Tel: 905332176828 E-mail: ekucuktulu@yahoo.com

Received February 28, 2018; Accepted March 05, 2018; Published March 08 2018

Citation: Kucuktulu E, Yurekli AF, Bilcan E, Sisecioglu MS, Topbas M, et al. (2018) Comparison of Unblocked, Directionally Blocked and Full Blocked Plans in Helical Tomotherapy for Breast Cancer Radiotherapy. J Nucl Med Radiat Ther 9: 358. doi: 10.4172/2155-9619.1000358

Copyright: (c) 2018 Kucuktulu E, et al. This is an open-access article distributed under the terms of the Creative Commons Attribution License, which permits unrestricted use, distribution, and reproduction in any medium, provided the original author and source are credited. 
Citation: Kucuktulu E, Yurekli AF, Bilcan E, Sisecioglu MS, Topbas M, et al. (2018) Comparison of Unblocked, Directionally Blocked and Full Blocked Plans in Helical Tomotherapy for Breast Cancer Radiotherapy. J Nucl Med Radiat Ther 9: 358. doi: 10.4172/2155-9619.1000358

surgery and in 16 patients Modified radical mastectomy was the surgical treatment preferred. According to our standard treatment protocol all patients underwent virtual simulation in supine position on a breast board, with ipsilateral arm extended above the head and the head turned away from the target breast. Radio opaque wires delineated superior, inferior, medial and lateral edges. The simulation was carried out on a Tomocon workstation using a non-contrast CT scan obtained in $3 \mathrm{~mm}$ slice range where target volumes and critical structures were defined. The whole breast or chest wall, axilla and supraclavicular field (SCF) clinical target volume (CTV), organs at risks (OARs) contoured were both lungs, contralateral breast and heart. The subsequent planning target volume (PTV) was generated by adding a $5 \mathrm{~mm}$ margin around the CTV but confined to the interior of the patient's outer contours reduced by $5 \mathrm{~mm}$. In all 20 patients the contours drawn in Tomocon workstation were transferred to Hi-Art planning system (TomoTherapy Inc, Madison WT). Three different therapy plans were designed: Non-blocked, directionally blocked and full blocked for contralateral breast. In tomotherapy planning systems 3 major factors are described: Field width, pith and modulation factor. The longitudinal field width is described as the fan beam width in craniocaudal direction. Pith is the ratio between the table motion and area with per gantry rotation. The modulation factor is the ratio between the most intense bundle intensity and the mean intensity of the whole bundles. In our study the field with was $5.02 \mathrm{~cm}$, pitch was 0.287 and the modulation factor was 2. In all TOMO plans the breast or chest wall, axilla and supraclavicular fossa should receive a total dose of $50 \mathrm{~Gy}$ in 25 fractions. Four of the patients received an additional boost of $10 \mathrm{~Gy}$ to the tumor bed. In all 3 therapy methods PTV $97 \%$ (47.5 Gy) and 107\% (53.5 Gy) were evaluated. (V95\%-V107\%) For all patients 3 plans with different methods were designed. These methods were nonblocked, directionally blocked and full blocked methods. In non-blocked method only dose restrictions were used. In directionally blocked method radiation is not delivered in single direction when the gantry sees the contralateral breast. In full blocked method, in every angle that the gantry sees the contralateral breast radiation is not delivered (Figure 1).

Homogenity Index (HI) and Conformity Number (CN) were calculated for all 3 planning methods.

$\mathrm{HI}$ is calculated as follows:

$$
\begin{aligned}
& H I=\frac{D_{2}-D_{98}}{D_{p}} \times 100 \\
& D_{2}=\text { Dose received by } 2 \% \text { of target volume } \\
& D_{98}=\text { Dose received by } 98 \% \text { of target volume } \\
& D_{p}=\text { Dose prescribed }
\end{aligned}
$$

$\mathrm{CN}$ is calculated as follows:

$C N=\frac{T V_{R I}}{T V} \times \frac{T V_{R I}}{V_{R I}}$

$C N=$ Conformity Number

$T V_{R I}=$ Target volume covered by the $95 \%$ Isodose line

$T V=$ Target Volume

$V_{R I}=$ Reference Isodose Volume

\section{Statistical analysis}

For comparisons in heart directional and full blocked plans Friedman test (post hoc Benferroni correction Wilcoxon test) was used. For other comparisons Repeated Measures ANOVA (post hoc Benferroni test) was used. Differences were considered significant if $\mathrm{p}<0.001$.

The study has been approved by Ethical Consul of Kanuni Research and Training Hospital.

\section{Results}

A typical dose distribution of three plans not only MRM but also BCS is displayed in Figures 2a-2f. The V95\% was more than 99\% in all plans. V107\% did not show significant difference among the three plans.

The mean CN were 0.95 (0.93-0.96), 0.95 (0.93-0.97) and 0.95 (0.920.96 ) for unblocked, directionally blocked and full blocked respectively. (Table 1) The mean HI were 7.51 (4.93-10.23) for unblocked, 7.45 (4.71-9.56) for directionally blocked and 8.87 (6.41-11.97) for full blocked plans. The difference was statistically significant.

The mean Lung V5 values were 98\% (79-100\%) in unblocked group and it was $98 \%$ (91-100\%) in directionally blocked group while

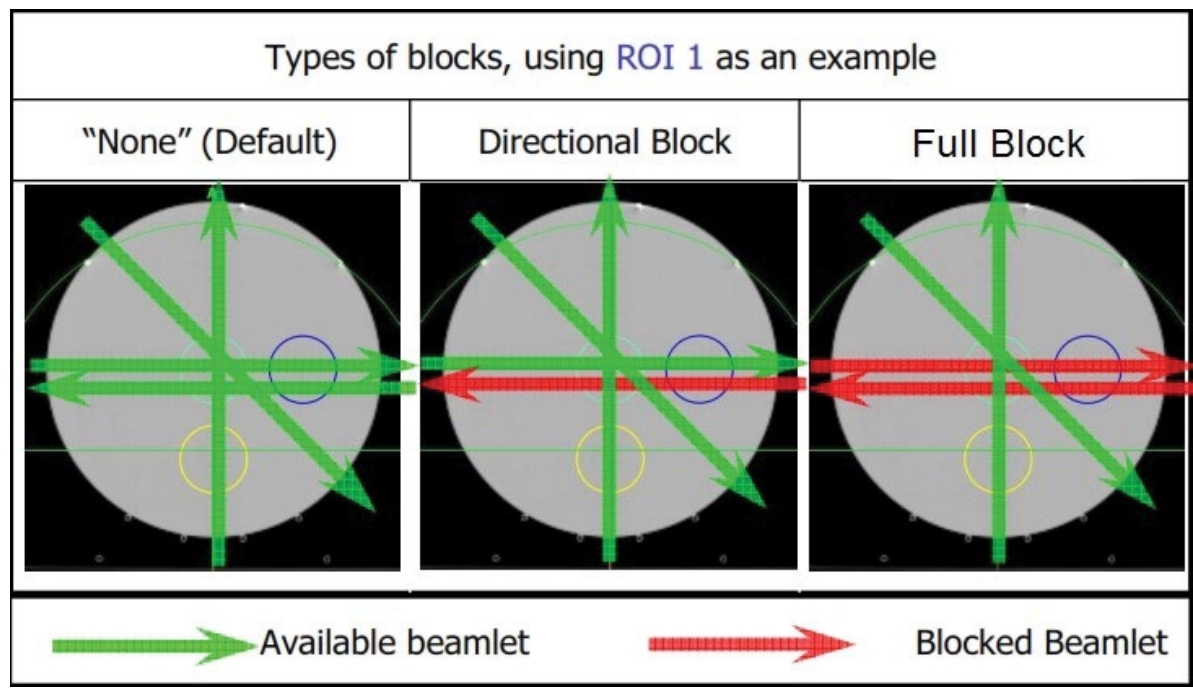

Figure 1: Methods of dose delivery. 
Citation: Kucuktulu E, Yurekli AF, Bilcan E, Sisecioglu MS, Topbas M, et al. (2018) Comparison of Unblocked, Directionally Blocked and Full Blocked Plans in Helical Tomotherapy for Breast Cancer Radiotherapy. J Nucl Med Radiat Ther 9: 358. doi: 10.4172/2155-9619.1000358
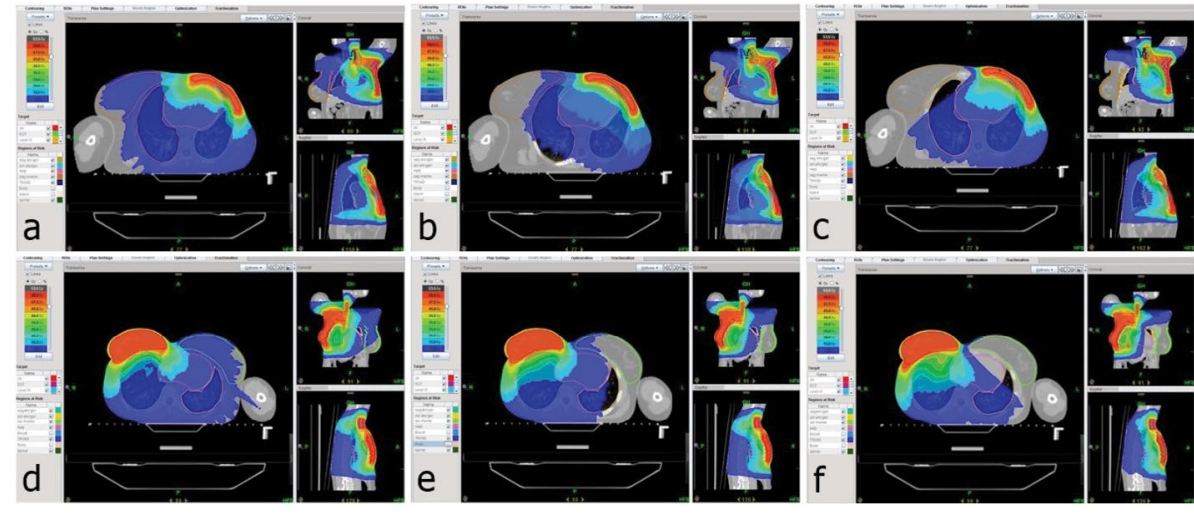

Figure 2: Radiotherapy plans.

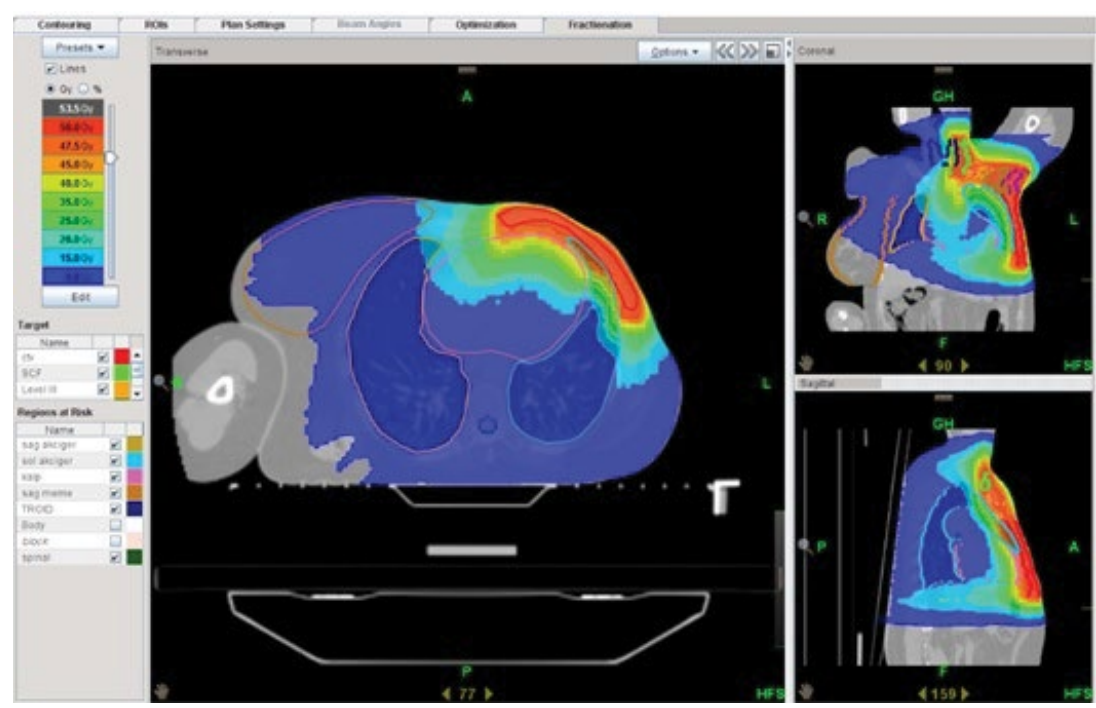

Figure 2a: Postmastectomy,nonblocked. (MU: 4028 Beam-on time 238.8 sn).

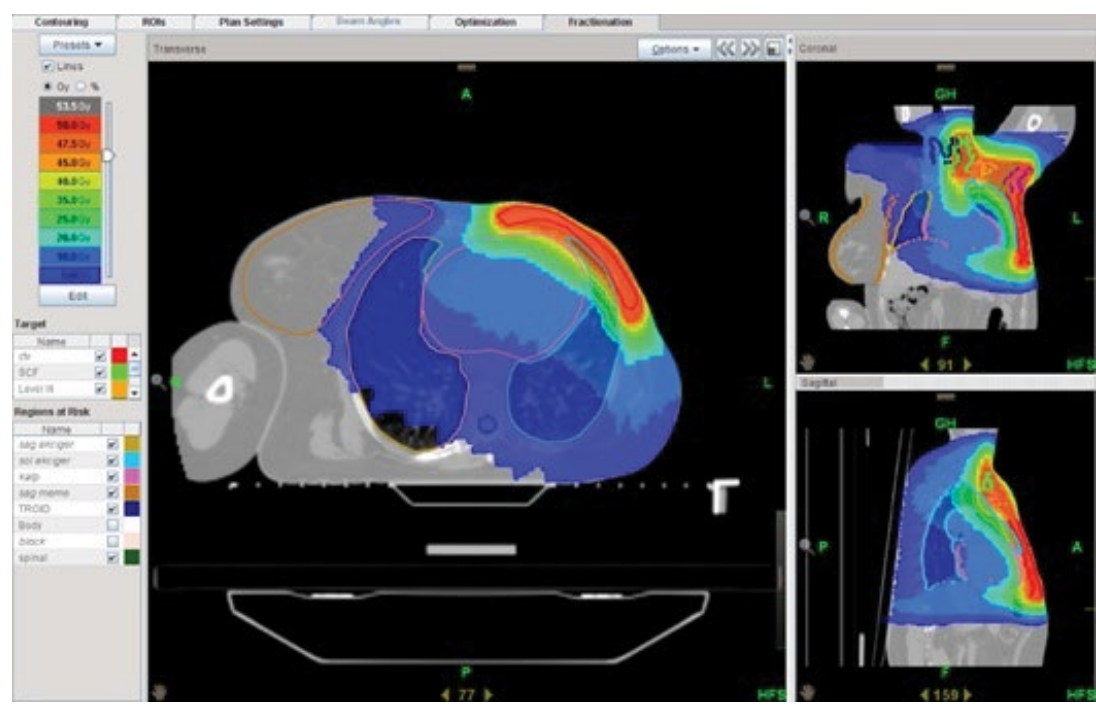

Figure 2b: Postmastectomy, directionally blocked (MU: 5466, Beam-on time 381.5sn). 
Citation: Kucuktulu E, Yurekli AF, Bilcan E, Sisecioglu MS, Topbas M, et al. (2018) Comparison of Unblocked, Directionally Blocked and Full Blocked Plans in Helical Tomotherapy for Breast Cancer Radiotherapy. J Nucl Med Radiat Ther 9: 358. doi: 10.4172/2155-9619.1000358

Page 4 of 7

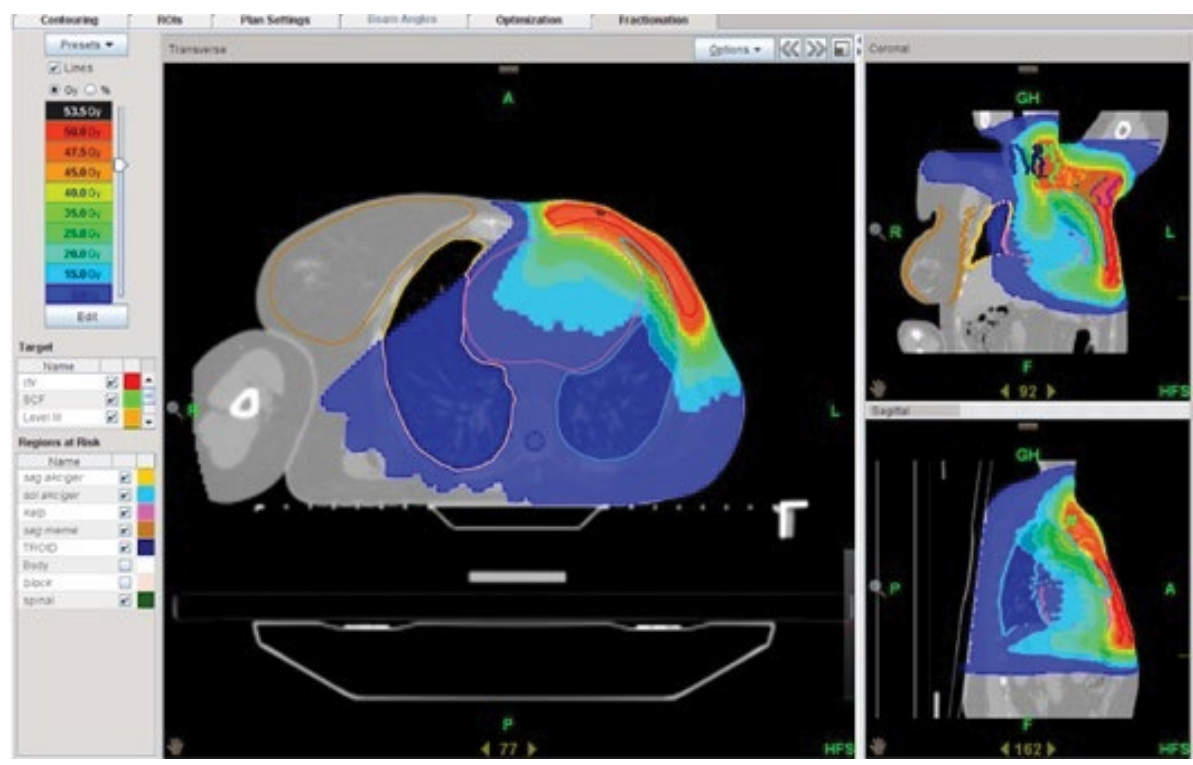

Figure 2c: Postmastectomy, full blocked. (MU: 6042 Beam-on time: 420.8 sn).

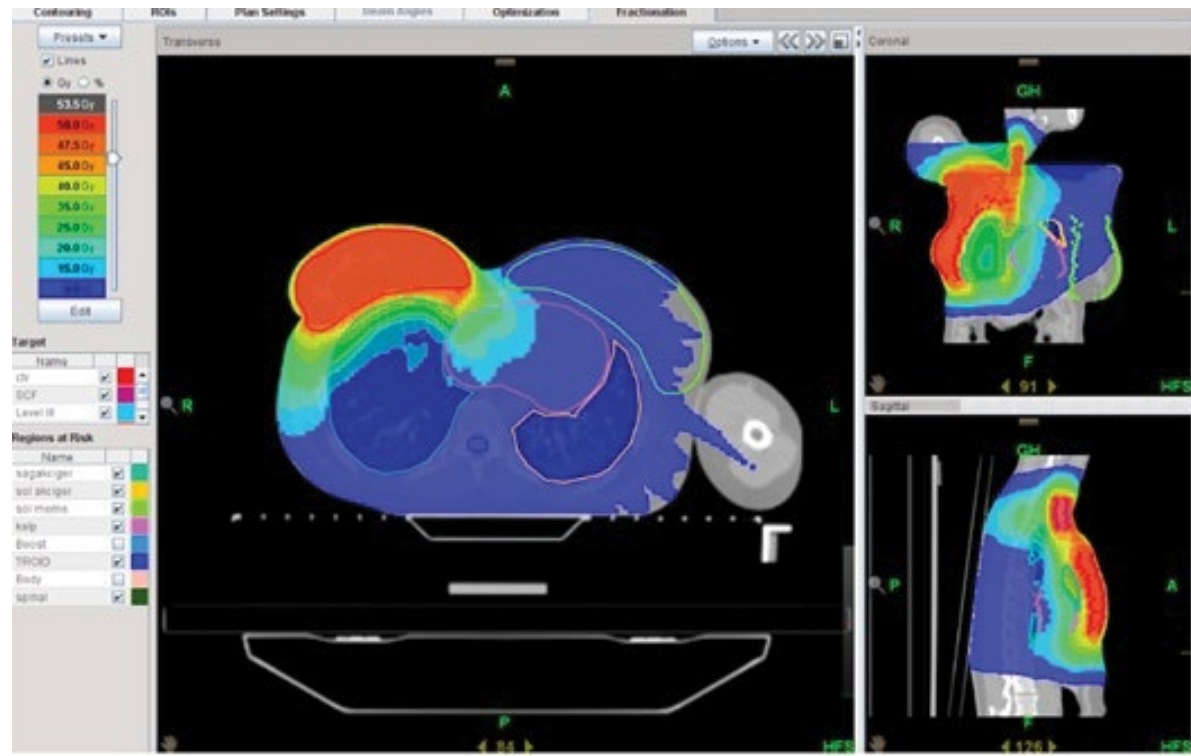

Figure 2d: Post breast conserving surgery, nonblocked. (MU: 3815 Beam-on time: 269.4 sn).

it was $93 \%$ (89-97\%) in full blocked group. The full blocked group was showed statistically significant difference $(\mathrm{p}<0.001)$ comparing to other groups. The mean V20 values were $16 \%$ (7-23\%), 20\% (17-24\%) and $22 \%(18-28 \%)$ in unblocked, directionally blocked and full blocked groups respectively. The difference was statistically significant. The mean Heart V5 value $100 \%$ (99-100\%) in unblocked group, and it was $99 \%(85-100 \%)$ in directionally blocked group while it was $92 \%$ (73$100 \%)$ in full blocked group. The difference was statistically significant $(\mathrm{p}<0.001)$. The mean V30 values were $11 \%(2-25 \%), 6 \%(0-12 \%)$ and $9 \%$ (1-22\%) in unblocked, directionally blocked and full blocked groups respectively. The difference was statistically significant $(p<0.001)$ in favor of directionally blocked group. When the mean radiation dose received by the contralateral breast was evaluated we found that the mean doses received were $6.24 \mathrm{~Gy}(4.50-8.74 \mathrm{~Gy})$ in unblocked group 3.85 Gy $(2.08-5.81 \mathrm{~Gy})$ in directionally blocked group and $0.99 \mathrm{~Gy}$ (0.69-1.39 Gy) in full blocked group. The difference was statistically significant $(\mathrm{p}<0.001)$ (Table 2$)$.

The mean beam-on time were $261.6 \mathrm{~s}$ (range 237.6-318.2) for unblocked, $277.9 \mathrm{~s}$ (range 237.6-338.5) for directionally blocked and $314.2 \mathrm{~s}$ (range 272.6-429.1) full blocked group. The mean numbers of MUs were 3712 (3353-4536) for unblocked, 3951 (3357-4834) for directionally blocked and 4506 (3866-6168) for full blocked group. In full blocked group both beam on time and MU values were higher. 
Citation: Kucuktulu E, Yurekli AF, Bilcan E, Sisecioglu MS, Topbas M, et al. (2018) Comparison of Unblocked, Directionally Blocked and Full Blocked Plans in Helical Tomotherapy for Breast Cancer Radiotherapy. J Nucl Med Radiat Ther 9: 358. doi: 10.4172/2155-9619.1000358

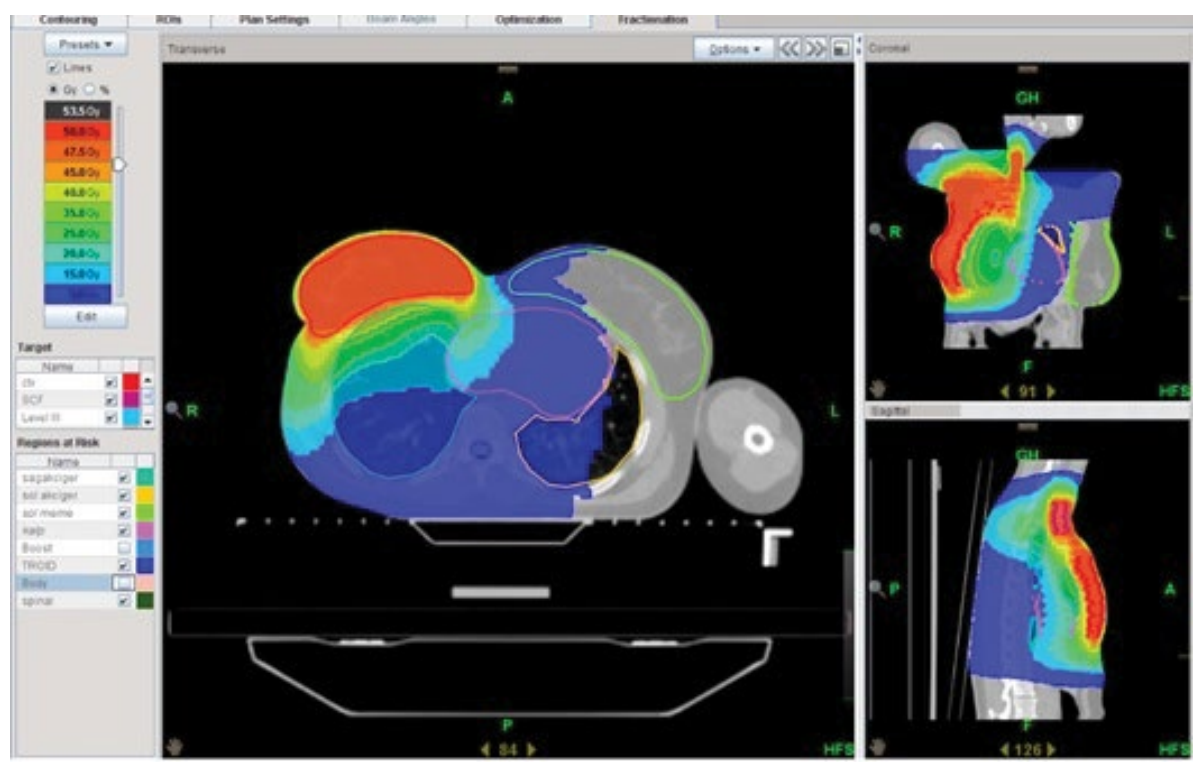

Figure 2e: Post breast conserving surgery, directionally blocked. (MU: 4402 Beam-on time: $309.4 \mathrm{sn}$ ).

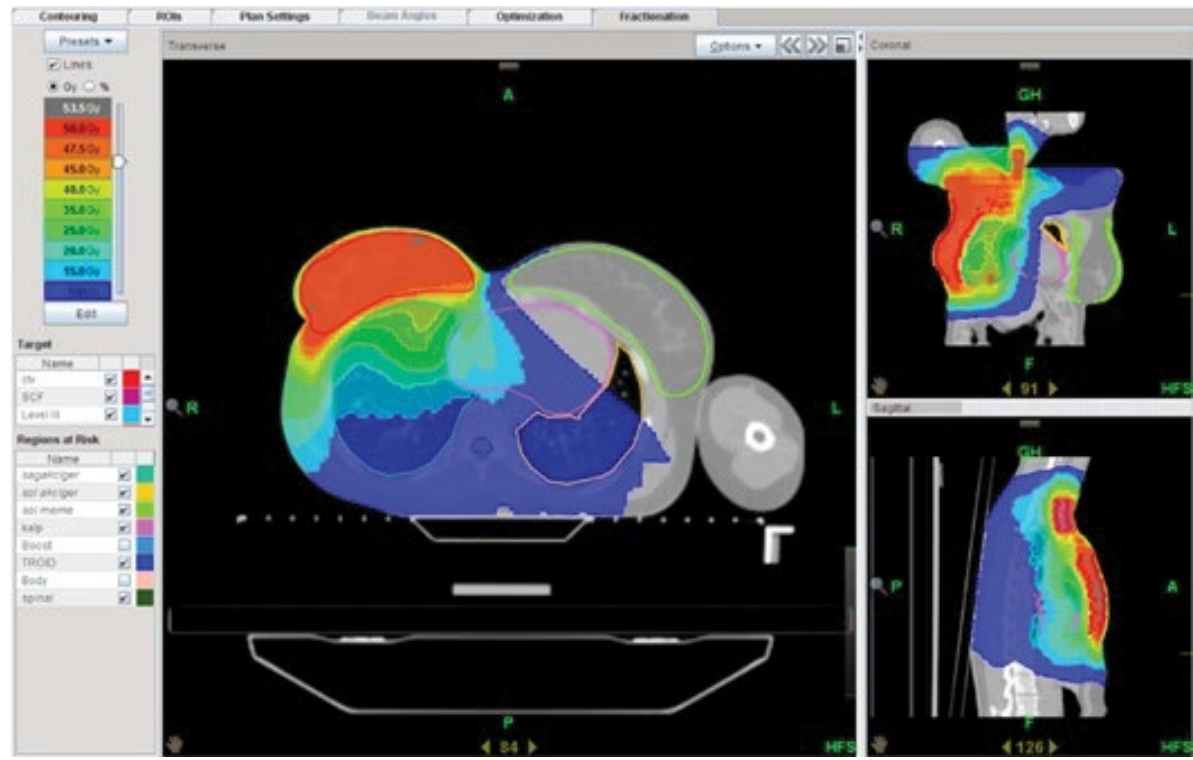

Figure 2f: Post breast conserving surgery, full blocked. (MU: 6163 Beam-on time: $429.1 \mathrm{sn}$ ).

\begin{tabular}{|r|c|c|c|c|}
\hline & Unblocked & Directionally blocked & Full blocked & p value \\
\hline Lung (\%) & \multicolumn{5}{|l|}{} \\
\hline V5 & 98 & 98 & 93 & \multirow{2}{*}{ p $<0.001$} \\
\hline V20 & 16 & 20 & 22 & \\
\hline V35 & 100 & 99 & 92 & \multirow{2}{*}{ p $<0.001$} \\
\hline
\end{tabular}

Contralateral Breast (Gy)

\begin{tabular}{|l|l|l|l|}
6.24 & 3.85 & 0.99 & $p<0.001$ \\
\hline
\end{tabular}

Table 1: Results of three different planning methods.

\begin{tabular}{|l|c|c|c|c|}
\hline & Unblocked & $\begin{array}{c}\text { Directionally } \\
\text { blocked }\end{array}$ & Full blocked & p value \\
\hline $\begin{array}{l}\text { Conformity Number } \\
\text { Mean (min-max) }\end{array}$ & $\begin{array}{c}0.95(0.93- \\
0.96)\end{array}$ & $\begin{array}{c}0.95(0.93- \\
0.97)\end{array}$ & $\begin{array}{c}0.95(0.92- \\
0.96)\end{array}$ & NS \\
\hline $\begin{array}{l}\text { Homogenity Index } \\
\text { Mean (min-max) }\end{array}$ & $\begin{array}{c}7.51(4.93- \\
10.23)\end{array}$ & $\begin{array}{c}7.45(4.71- \\
9.56)\end{array}$ & $\begin{array}{c}8.87(6.41- \\
11.97)\end{array}$ & $\mathrm{P}<0.05$ \\
\hline
\end{tabular}

Table 2: $\mathrm{HI}$ and $\mathrm{CN}$ for 3 planning methods.

\section{Discussion}

HT manufactured and presented for commercial use with the name of Tomotherapy Hi-Art System in 2002 after it had been developed in University of Wisconsin. HT is the technique of preference for irradiation of the thoracic wall and the regional lymph 
nodes particularly in left breast after mastectomy and axillar dissection, especially in patients with anatomies that may result too much heart and lung radiation doses if otherwise irradiated with conventional methods, in presence of breast implants or in cases of challenging regional field irradiation [9]. In a study of Ashenafi et al comparing HT and conventional mixed-beam plans, they reported that HT planning resulted in better dose homogeneity in chest wall and internal mammarian nodes, PTV was significantly better and resulted larger volumes of low dose for the lung and contralateral breast. Improved dose homogeneity in target volumes offers improved post therapy cosmesis. There is a slight improvement in the V20 of the ipsilateral lung with the HT technique [10]. In our study the V5 values were $98 \%$ and $100 \%$ for lung and heart respectively. The V20 values of lung and the V30 values of heart were in accordance with Radiation Therapy Oncology Group (RTOG) 0413 protocol guidelines.

For this reason to protect the contralateral breast heart and lung, a new mode called 'Tomodirect' which was previously named 'Topotherapy' has been added to HT. This mode uses a fixed gantry angle, similar to conventional radiotherapy that most clinicians are familiar with and it is available for clinical use since April 2010 This combination of two delivery modes gives us more freedom and represents a new treatment option for very highly conformal radiotherapy for breast treatment.

Comparison of coplanar accelerated partial breast topotherapy with Linac based non-coplanar 3D conformal radiotherapy and IMRT dosimetry in partial left breast irradiation, illustrates equivalent target conformity and uniformity [11]. Dosimetric comparison of two field topotherapy with HT for the delivery of whole breast radiotherapy showed improved target dose homogeneity and conformality in comparison to with topotherapy. However, topotherapy resulted into reduced amounts of the heart and ipsilateral lung receiving low doses while still maintaining adequate target uniformity. The higher number of the fields, up to five for the topotherapy plans, was correlated with increase conformality [12]. The topotherapy plans were slightly less homogeneous and had a slightly greater target dose maximum comparing to helical plans [13]. Han et al in a study comparing five different radiotherapy modalities including Tomo HDA reported that Tomotherapy plan provided plan quality comparable to the IMRT plan and posed the lowest total lifetime attributable risk to neighboring organs They also reported that MU values were longer in Tomo plans. We also found that in full blocked plans the doses dropped in controlateral breast but MU is prolonged. As Han et al reported in their study MUs of Tomo cannot be explicitly compared to MUs of traditional LINAC [14]. In our study CN was better comparing to that of Tomo HDA plans reported by Han et al.

HT has the advantage that the beam from all gantry angles causes delivery of low doses to coplanar normal tissues, most of which would have received only a scatter dose with the conventional techniques. During HT treatment planning optimization, critical structures can be designated as a blocked region to prevent any beamlet from passing through the structure, or can be directionally and full blocked, thus inhibiting any entry of the beamlets to the structure, but still allowing their exit. This sort of blocking reduces treatment beam angles by minimizing dose to the contralateral structures; however, it reduces the potential conformality and sparing of the ipsilateral organs at risk [9]. Reduction of the contralateral structures doses for HT plans causes an increase in treatment time [12]. In our study when HT plans were done in unblocked method the contralateral breast doses were 3.5-8.7 Gy. We tried to reduce the dose with directionally and full blocked methods. We did not use directionally or full blocked methods because V20 for lung and V30 for heart were in acceptable dose levels when unblocked plans were used. For both organs V5 values were found quite high and these results were in accordance with the findings of the literature. When directionally blocked and full blocked methods were used to reduce V5 values for heart and lung, the beam-on time and MU increased in full blocked method we observed further increases in these parameters. For these reasons we used blocked methods only for contralateral breasts to provide dose homogeneity and prevent greater target dose maximums. We minimized the contralateral breast doses to minimum with the use of full blocked method. When full blocked method was used the V20 doses for lung increased. Although V20 doses of lung was still in acceptable levels these increments was statistically significant.

The current technology advances in radiotherapy and further improvements in treatment outcomes made reduction of early and late radiotherapy toxicities an emerging challenge. After the improvements on early diagnosis and treatment modalities, the breast cancer survivors currently live longer and late treatment sequel, such as secondary cancers became more important that need to be minimized. [15] The dosimetric tradeoff of "a lot to a little or a little to a lot" has been a subject of debate for several years, especially since the implementation of IMRT techniques [7]. Some may raise concerns regarding the risks of second cancers in normal tissues irradiated to low dose [16]. The use of IMRT may significantly increase the doses to normal tissues [17]. The total body dose is expected to be two to three times higher, predominantly owing to the increased number of beams used, as well increased leakage radiation (resulting from increased "beam on" time) [15].

Boice et al. reported 1.19 relative risk of secondary contralateral breast cancer after previous WBRT (estimated average radiation dose: 2.82 Gy, Dmax 7.1 Gy), Thus, a dose range between 4-5 Gy to the contralateral breast might be considered sufficiently safe in terms of radiogenic second malignancies [18]. In the literature six randomized studies compared the use of radiotherapy with no radiotherapy one showed increased risk in the irradiated group, two a trend toward increase, and three a decreased incidence of contralateral breast cancer [17]. As the carcinogenic effect of radiation on breast tissue was shown in many studies, it is reasonable to make every effort to limit the doses of incidental irradiation $[19,20]$. IMRT may therefore increase the incidence of second tumors in 10 year survivors; this will predominantly include tumors resulting from low exposure, such as leukemia and carcinomas [21]. Therefore it is necessary to find a new method to provide minimum dose levels and dose homogeneity for heart and lung as for contralateral breast, without increasing beamon time and MU. Although Tomodirect provides these conditions it can provide less dose homogeneity. Three large studies confirmed a significantly increased incidence of ipsilateral lung cancer after irradiation for breast cancer [22].

HT is currently one of the most sophisticated forms of IMRT implemented in clinical practice. Since it is relatively a new technique the effects on survival and local control, as well as long term toxicities is not yet clear [23-25] Especially in the presence of breast implants that requires challenging regional field irradiation, HT provides quite good dose distribution and enables implant protection. Considering achievements in breast surgery, we propose that further efforts needed to reduce low dose volumes to normal tissues, meanwhile when using directionally and full blocked plans all parameters should be considered 
Citation: Kucuktulu E, Yurekli AF, Bilcan E, Sisecioglu MS, Topbas M, et al. (2018) Comparison of Unblocked, Directionally Blocked and Full Blocked Plans in Helical Tomotherapy for Breast Cancer Radiotherapy. J Nucl Med Radiat Ther 9: 358. doi: 10.4172/2155-9619.1000358

together. This study can pioneer further studies comparing Tomo HDA and HT with various block plans.

\section{Conclusions}

The Tomotherapy based IMRT plans provide better prescribed doses to the target in expense of increased volume of lower dose of the ipsilateral lung heart and contralateral breast. To reduce this lower dose volume topotherapy method has recently been developed. When two field topotherapy plans with tangent like gantry angles is done, it spares the ipsilateral lung and heart and contralateral breast better but reduces dose conformation to the target volume. In HT plans it has been observed that in directionally or full blocked plans for contralateral breast, the contralateral breast is protected but directionally or full blocked plans are also required for lung and heart to protect them which prolongs treatment times and creates dose homogeneity problems. Especially in case of bilateral mastectomy, using blocks for lung and heart low dose volumes can be reduced. New studies are required on directionally and full blocked treatment plans to reduce the treatment time. Until then case based evaluations are crucial.

\section{Competing Interests}

The authors declare that they have no competing interests.

\section{References}

1. Dogan N, Cuttino L, Lloyd R, Bump EA, Arthur DW (2007) Optimized dose coverage of regional lymph nodes in breast cancer: The role of intensitymodulated radiotherapy. Int J Radiat Oncol Biol Phys 68: 1238-1250.

2. Evans PM, Donovan EM, Partridge M, Childs PJ, Convery DJ, et al. (2000) The delivery of intensity modulated radiotherapy to the breast using multiple static fields. Radiother Oncol 57: 79-89.

3. Johansson J, Isacsson U, Lindman H, Montelius A, Glimelius B (2002) Nodepositive left-sided breast cancer patients after breast-conserving surgery: Potential outcomes of radiotherapy modalities and techniques. Radiother Oncol 65: 89-98.

4. Popescu CC, Olivotto I, Patenaude V, Wai E, Beckham WA (2006) Inverseplanned, dynamic, multi-beam, intensity-modulated radiation therapy (IMRT): A promising technique when target volume is the left breast and internal mammary lymph nodes. Med Dosim 31: 283-291.

5. Krueger EA, Fraass BA, McShan DL, Marsh R, Pierce LJ (2003) Potential gains for irradiation of chest wall and regional nodes with intensity modulated radiotherapy. Int J Radiat Oncol Biol Phys 56: 1023-1037.

6. Thilmann C, Sroka-Perez G, Krempien R, Hoess A, Wannenmacher M, et al. (2004) Inversely planned intensity modulated radiotherapy of the breast including the internal mammary chain: A plan comparison study. Technol Cancer Res Treat 3: 69-75.

7. Caudrelier JM, Morgan SC, Montgomery L, Lacelle M, Nyiri B (2009) Helical tomotherapy for locoregional irradiation including the internal mammary chain in left-sided breast cancer: Dosimetric evaluation. Radiother Oncol 90: 99-105.

8. Caudell JJ, De Los Santos JF, Keene KS, Fiveash JB, Wang W (2007) A dosimetric comparison of electronic compensation, conventional intensity modulated radiotherapy and tomotherapy in patients with early-stage carcinoma of the left breast. Int J Radiat Oncol Biol Phys 68: 1505-1511.
9. Haydaroglu A, Ozyigit G (2013) Principles and practice of modern radiotherapy techniques in breast cancer, New York, USA.

10. Ashenafi M, Boyd RA, Lee TK, Lo KK, Gibbons JP, et al. (2010) Feasibility of post-mastectomy treatment with helical TomoTherapy. Int J Radiat Oncol Biol Phys 77: 836-842.

11. McIntosh A, Read PW, Khandelwal SR, Arthur DW, Turner AB, et al. (2008) Evaluation of coplanar partial left breast irradiation using tomotherapy-based topotherapy. Int J Radiat Oncol Biol Phys 71: 603-610.

12. Gonzalez VJ, Buchholz DJ, Langen KM, Olivera GH, Chauhan B, et al. (2006) Evaluation of two TomoTherapy-based techniques for the delivery of wholebreast Intensity modulated radiation therapy. Int J Radiat Oncol Biol Phys 65 284-290.

13. Langen KM, Buchholz DJ, Burch DR, Burkavage R, Limaye AU, et al. (2008) Investigation of accelerated partial breast patient alignment and treatment with helical tomotherapy unit. Int J Radiat Oncol Biol Phys 70: 1272-1280.

14. Han EY, Paudel N, Sung J, Yoon M, Chung WK, et al. (2016) Estimation of the risk of secondary malignancy arising from whole-breast irradiation: Comparison of five radiotherapy modalities, including TomoHD. Oncotarget 7: 22960-22969.

15. Nagai A, Shibamoto Y, Yoshida M, Inoda K, Kikuchi $Y$ (2017) Intensitymodulated radiotherapy using two static ports of tomotherapy for breast cancer after conservative surgery: Dosimetric comparison with other treatment methods and 3-year clinical results. J Radiat Res 58: 529-536.

16. Roychoudhuri R, Evans H, Robinson D, Møller H (2004) Radiation-induced malignancies following radiotherapy for breast cancer. $\mathrm{Br} \mathrm{J}$ Cancer 91: 868872.

17. Hall EJ, Wuu CS (2003) Radiation-induced second cancers: The impact of 3D-CRT and IMRT. Int J Radiat Oncol Biol Phys 56: 83-88.

18. Unnithan J, Macklis RM (2001) Contralateral breast cancer risk. Radiather Oncol 60: 239-246.

19. Boice JD Jr, Harvey EB, Blettner M, Stovall M, Flannery JT (1992) Cancer in the contralateral breast after radiotherapy for breast cancer. New Engl $\mathrm{J} \mathrm{Med}$ 326: 1243-1251.

20. Houghton J, Baum M, Hayybittle JL (1994) Role of radiotherapy following tota mastectomy in patients with early breast cancer. The closed trials working party of the CRC breast cancer trials group. World J Surg 18: 117-122.

21. Fowble B, Hanlon A, Freedman G, Nicolaou N, Anderson P (2001) Second cancers after conservative surgery and radiation for stage I-II breast cancer: Identifying a subset of women at increased risk. Int J Radiat Oncol Biol Phys 51: $679-690$

22. Neugut Al, Murray T, Santos J, Amols H, Hayes MK, et al. (1994) Increased risk of lung cancer after breast cancer radiation therapy in cigarette smokers. Cancer 73: 1615-1620

23. Zablotska LB, Neugut Al (2003) Lung carcinoma after radiation therapy in women treated with lumpectomy or mastectomy for primary breast carcinoma. Cancer 97: 1404-1411

24. George R, Keall PJ, Kini VR, Vedam SS, Siebers JV, et al. (2003) Quantifying the effect of intrafraction motion during breast IMRT planning and dose delivery. Med Phys 30: 552-562.

25. Ramsey CR, Seibert RM, Robison B, Mitchell M (2007) Helical tomotherapy superficial dose measurements. Med Phys 34: 3286-3293. 